\title{
Recent results from MICE on multiple Coulomb scattering and energy loss
}

John Columba Nugent*

University of Glasgow

E-mail: john.nugenteglasgow.ac.uk

Paul Soler

University of Glasgow

E-mail: paul.soler@glasgow.ac.uk

\section{Ryan Bayes}

Laurentian University

E-mail: RBayes@laurentian.ca

\begin{abstract}
Multiple coulomb scattering and energy loss are well known phenomena experienced by charged particles as they traverse a material. However, from recent measurements by the MuScat collaboration, it is known that the available simulation codes (GEANT4, for example) overestimate the scattering of muons in low $\mathrm{Z}$ materials. This is of particular interest to the Muon Ionization Cooling Experiment (MICE) collaboration which has the goal of measuring the reduction of the emittance of a muon beam induced by energy loss in low $\mathrm{Z}$ absorbers. MICE took data without magnetic field suitable for multiple scattering measurements in the spring of 2016 using a lithium hydride absorber. The scattering data are compared with the predictions of various models, including the default GEANT4 model.
\end{abstract}

The 19th International Workshop on Neutrinos from Accelerators - NUFACT2017

25-30 September, 2017

Uppsala University, Uppsala, Sweden

${ }^{*}$ Speaker. 


\section{Introduction}

Results from atmospheric neutrinos at Super-Kamiokande [1] and from solar neutrinos at the Sudbury Neutrino Observatory [2] conclusively demonstrated that neutrinos have a non-zero mass and oscillate between different flavours. A facility promising precision measurement of neutrino oscillations parameters is the Neutrino Factory [3], where neutrinos would be produced via muon decay rings. Before the muons are injected into the storage ring the phase-space volume of the beam must be reduced. The only cooling technique which can act within the lifetime of the muon is ionization cooling and has shown in simulation to reduce the phase-space volume of the beam by a factor of 100,000 [4, 5, 6]. MICE Step IV is current taking data to provide the first measurement of ionization cooling. This demonstration is an essential part of the worldwide research effort towards building a Neutrino Factory. A Neutrino Factory is the only proposed facility with the capability to measure the $\mathrm{CP}$ violation phase, $\delta_{\mathrm{CP}}$, with $5^{\circ}$ accuracy.

\section{MICE Beam Line and Experiment}

The MICE experiment is located at the Rutherford Appleton Laboratory (RAL) in the UK and operates parasitically on the ISIS proton accelerator [7], producing beam for the newly built MICE Muon Beam (MMB) by the insertion of an internal pion-production target. MICE is a novel single particle experiment designed to perform high precision measurements of normalized emittance both upstream and downstream of the ionization cooling equipment. The MMB is composed of three quadrupole triplets, two dipole magnets, which select the momentum, and a decay solenoid (DS), which increases the number of muons in the beam. It consists of an Absorber Focus Coil (AFC) located between two measurement stations. These stations are composed of particle identification suites including a total of three time-of-flight detectors (TOFs) [8], two Cherenkov detectors (Ckova and Ckovb) [9], the KLOE-type sampling calorimeter (KL) [10] and the Electron Muon Ranger (EMR) [11]. Each station has a Tracker with five planes of scintillating fibres inside a 4 T Spectrometer Solenoid (SS) to measure track and momentum information $\left(x, y, p_{x}\right.$ and $\left.p_{y}\right)$, so as to reconstruct the emittance before and after cooling. In MICE Step IV the AFC module, which houses the liquid hydrogen or lithium hydride absorber within a focusing coil, is located between the two measurement stations.

\section{Overview of multiple Coulomb scattering}

The PDG recommends an approximate multiple scattering formula $[12,13]$, which is found to be accurate to approximately $11 \%$ :

$$
\theta_{0} \approx \frac{13.6 \mathrm{MeV}}{p_{\mu} \beta_{\mathrm{rel}}} \sqrt{\frac{\Delta z}{X_{0}}}\left[1+0.0038 \ln \left(\frac{\Delta z}{X_{0}}\right)\right],
$$

where $\theta_{0}$ is the rms width of the projected scattering angle distribution, $X_{0}$ is the radiation length of the material and $\Delta z$ is the thickness of the absorber, $p_{\mu}$ is the momentum of the muon and $\beta_{\text {rel }}=p_{\mu} c / E_{\mu}$, with $E_{\mu}$ its energy. From this an approximate cooling formula can be derived (ignoring the logarithmic term of equation 3.1), 


$$
\frac{d \varepsilon_{n}}{d z}=-\frac{\varepsilon_{n}}{E_{\mu} \beta_{\mathrm{rel}}^{2}}\left\langle\frac{d E_{\mu}}{d z}\right\rangle+\frac{\beta}{2 m_{\mu} \beta_{\mathrm{rel}}^{3}} \frac{(13.6 \mathrm{MeV})^{2}}{E_{\mu} X_{0}}
$$

where $\varepsilon_{n}$ is the normalised transverse (two-dimensional) emittance of the beam, $\beta$ is the betatron function, and $m_{\mu}$ the energy and mass of the muons [14]. Given that the goal of MICE is to measure the reduction in normalised emittance with $1 \%$ precision, which requires an absolute emittance measurement precision of $0.1 \%$, this approximate formula is not sufficient for the needs of MICE. This demands an accurate measurement of MCS for relevant low- $Z$ materials, such as liquid hydrogen and lithium hydride, where simulations are not in good agreement with data. The MUSCAT experiment carried out a measurement of muon scattering in low- $Z$ materials [15] and found significant differences between a number of models and the measured distributions.

The theory of Multiple Coulomb Scattering, developed by Rossi and Greisen [16] and Molière [17] considered the Rutherford scattering with a low angle cut-off

$$
\frac{\theta_{0}^{2}}{z}=16 \pi N_{A} \frac{Z^{2}}{A} r_{e}^{2}\left(\frac{m c}{p_{\mu} \beta}\right) \ln \left(196 Z^{-1 / 3}\left(\frac{Z}{A}^{1 / 6}\right)\right) \approx \frac{(21.2 \mathrm{MeV})^{2}}{p_{\mu}^{2} \beta^{2}} \frac{1}{X_{0}}
$$

where only interactions with the atomic nucleus are included, with a distribution proportional to $Z^{2}$, where $Z$ is the atomic number of the scattering material. Bethe [18] adapted the Molière theory to include atomic electron scattering, which implied a proportionality of $Z(Z+1)$ however this theory still assumed equal weight was given to both nuclear and atomic electron scattering. Early theories of MCS were reviewed by Scott [19]. Further modifications were made to the original theory by Lynch and Dahl to incorporate a path length dependance which resulted in the PDG formulate quoted in expression 3.1. Both the Molière and Bethe theories were compared to MCS data for a variety of absorbers in MUSCAT, and it was found that these theories did not describe low- $Z$ materials adequately.

Most particle physics simulations use GEANT4 [20] to evaluate particle interactions with matter. GEANT4 makes a parameterisation of the scattering distribtution for finite thickness of the material then proceeds stepwise through the simulated material calculating the contribution for each step. Multiple scattering in GEANT4 does not use a small angle approximation and relies on a Legendre polynomial expansion, where the default physics list evaluates the Urban cross-section [21, 22] for most particles and the Wentzel single-scattering cross-section for muons. This model works well for high- $Z$ materials but overestimates scattering for low- $Z$ materials. Alternative models which can overcome these shortcomings have been proposed, namely the Cobb-Carlisle model [14, 23] which samples directly from the Wentzel single-scattering cross-section and simulates all collisions with nuclei and electrons. This includes a cut-off for the nuclear cross-section and seperate contributions from the nuclear and atomic electron scattering

$$
\frac{\theta_{0}^{2}}{z}=8 \pi N_{A} \frac{Z^{2}}{A} r_{e}^{2}\left(\frac{m c}{p_{\mu} \beta}\left[\ln \left({\frac{\theta_{2}^{2}}{\theta_{1}}}^{2}+1\right)-1\right]+\frac{1}{Z}\left[\ln \left(\frac{\theta_{2}^{e 2}}{\theta_{1}^{e}}+1\right)-1\right]\right)
$$


Therefore, MICE will need to measure MCS for low- $Z$ materials, such as liquid hydrogen and lithium hydride, to perform an accurate measurement of ionisation cooling. The lithium hydride absorber has a thickness of $65 \mathrm{~mm}\left(6.7 \% X_{0}\right)$ where the lithium hydride composition is: $81 \%{ }^{6} \mathrm{Li}$, $4 \%{ }^{7} \mathrm{Li}, 14 \%{ }^{1} \mathrm{H}$ with traces of $\mathrm{C}, \mathrm{O}$, and $\mathrm{Ca}$.

\section{Scattering Data}
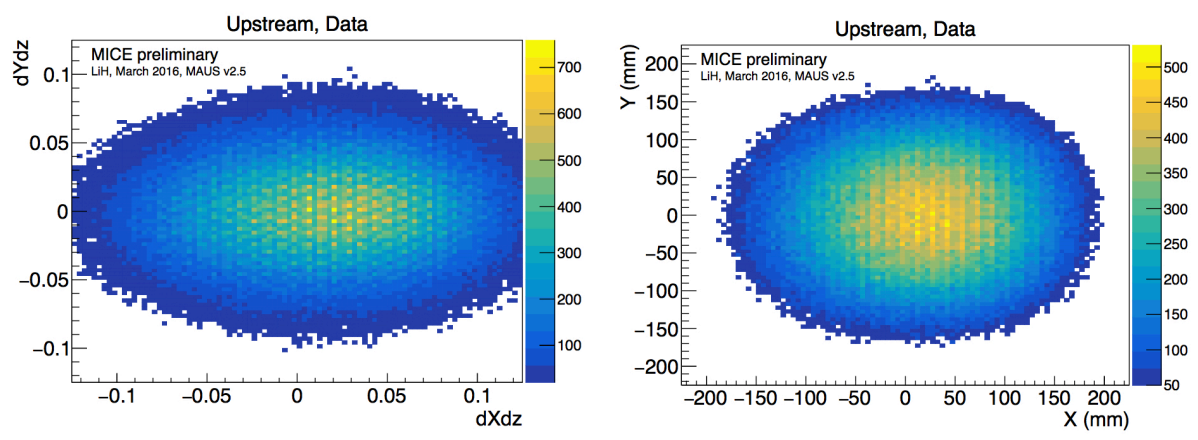

Figure 1: Upstream position distributions for a $200 \mathrm{MeV} / \mathrm{c}$ muon beam in the $\mathrm{LiH}$ data after particle selection.
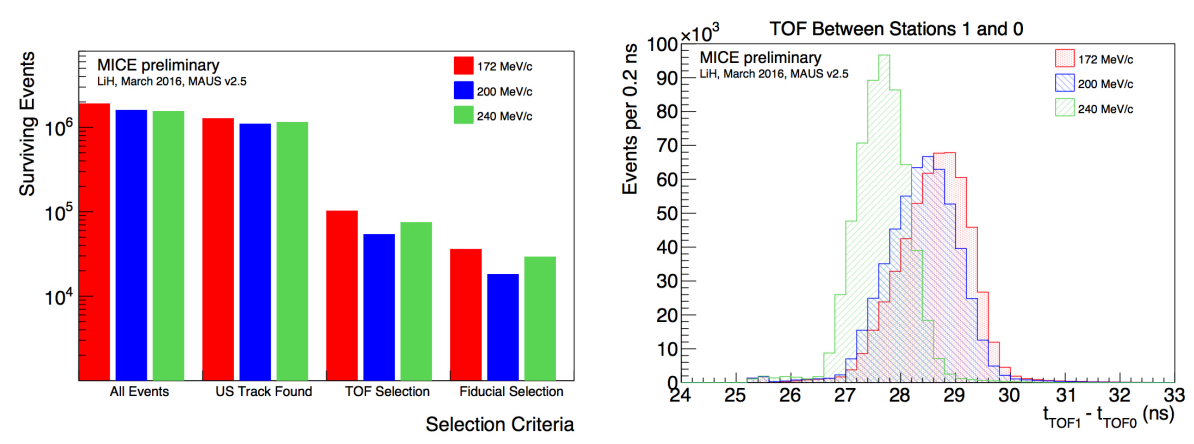

Figure 2: Left: The survival of muons after each stage of the selection. Right: The time of flight for muons between TOF0 and TOF1 for each of the nominal momnetum points that MICE will measure scattering at.

The MICE Muon Beam has been fully characterised [24] and has a pion contamination of less than $1.4 \%$ at $90 \%$ C.L. [25]. For the lithium hydride data the beam was operated in "muon mode" giving an almost pure muon beam at a variety of momenta. For this measurement a beam with a $3 \pi \mathrm{mm}$. rad emittance beam was selected. The lithium hydride MCS data taking period was during the 2015/04 ISIS user cycle from the 23rd of February until the 24th of March 2016. The volume between the absorber and the two trackers was filled with helium to minimise multiple scattering not due to the absorber. The measurements were carried out without a magnetic field either in the tracker volume or surrounding the absorber. During the 2015/04 data taking period, the channel was entirely empty when the lithium hydride was removed. Momentum measurements were carried out using the time-of-flight difference between TOF1 and TOF2. Only tracks that have hits in TOF1 in both planes, with one muon reconstructed in each event and within the time 
of flight window, are selected for the analysis. Tracks are projected downstream from the upstream tracker volume to the reference plane of the downstream tracker and must be within a $150 \mathrm{~mm}$ radius from the centre of the reference plane to be selected. The beam spot in the trackers after selection is shown in figure 1 and the population of muons after each selection is shown in figure 2.

\section{Momentum Correction}

The two MICE trackers can measure the momentum of muons up- and downstream of the absorber when both spectrometer solenoids are energised. When the solenoidal magnetic fields are present in the channel muons follow a helical trajectory from which a momentum measurement can be made. In the case where there are no magnetic field in the cooling channel no measurement of momentum can be made with the trackers. In this scenario a momentum measurement is made with the MICE time of flight system using the expression:

$$
p=\frac{m}{\sqrt{\frac{t_{12}^{2} c^{2}}{L^{2}}-1}}
$$

However this expression makes several assumptions, namely that the muons are on axis and undergo no energy loss between the TOF stations. To account for these effects a correction is applied to the momentum as reconstructed by the TOF system to reconstruct the exact momentum at the centre of the absorber. The calculation is an analytic expression which is the second order expansion of the Taylor series in $p / m c$ for the exact path of the muon between TOF stations. One caveat of this method is that constant energy loss is assumed. However even with this approximation good agreement between reconstructed momentum and that obtained from MC truth is shown in figure 3 .
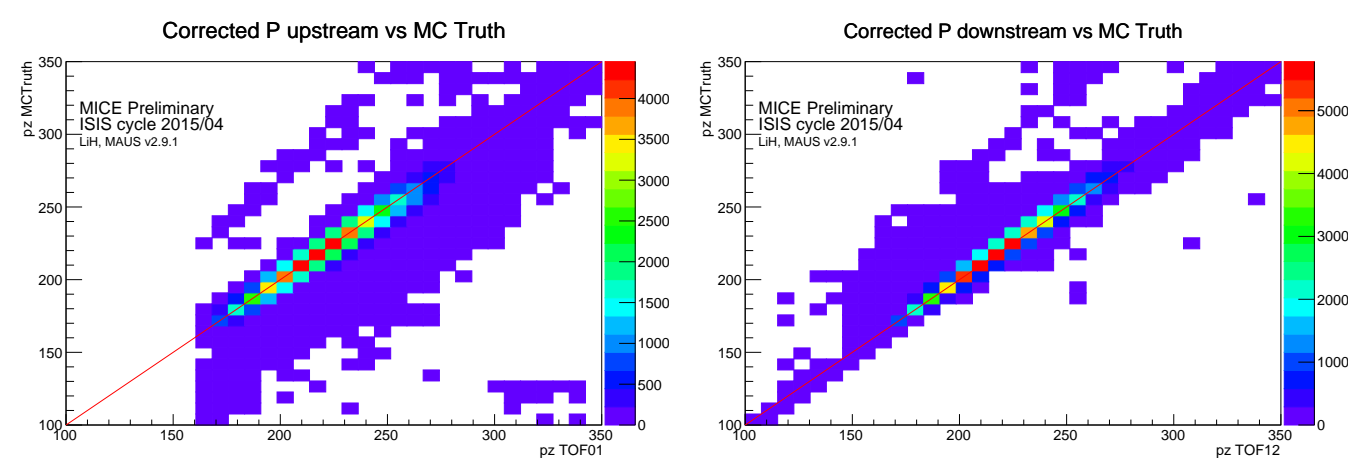

Figure 3: Figures showing the agreement between the momentum calculated by the correction up- (left) and downstream (right) and the momentum determined from MC truth.

\section{Tracker Acceptance}

The geometric acceptance of the scattering angles that can be measured by MICE are determined by the apertures of the cooling channel. The effect of this acceptance on the scattering 

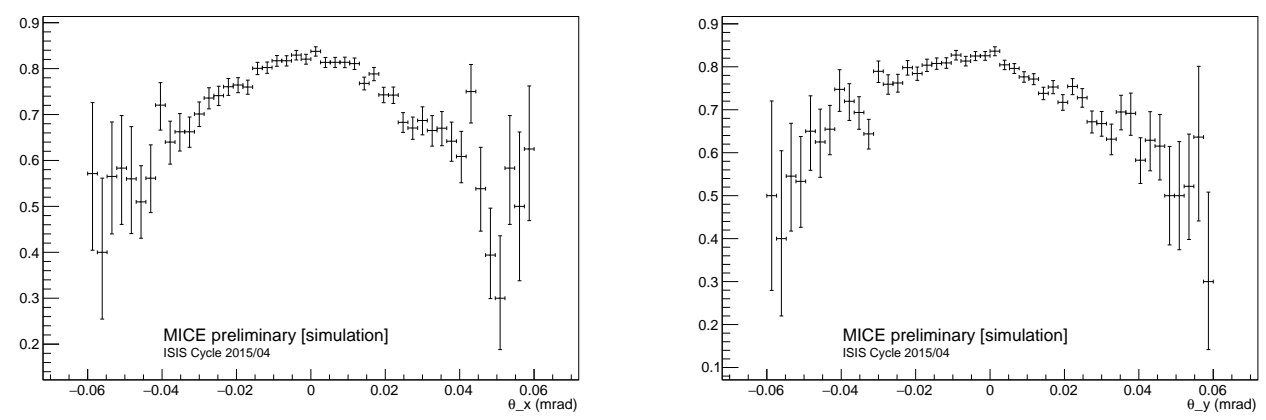

Figure 4: Figures showing the downstream tracker acceptance in $\theta_{x}$ (left) and $\theta_{y}$ (right).

distributions must be accounted for and is determined by considering Monte Carlo (MC) simulations of the MICE beam. In simulation both the number of tracks which are expected (i.e. seen in $\mathrm{MC}$ truth) and number of tracks reconstructed (i.e. seen in the reconstructed data) is known. The full analysis chain is then run, tracks are matched upstream and downstream, the selection is performed and the scattering angle is calculated. The downstream acceptance is then defined as

No. of tracks in $\theta$ bin MC Truth that are reconstructed

No. of tracks in $\theta$ bin MC Truth

It is assumed that the upstream efficiency is $100 \%$, as by construction if a track is never seen upstream then no scattering angle is ever measured. The measured acceptance is shown in figure 4.

\section{Deconvolution of Raw Scattering Data}
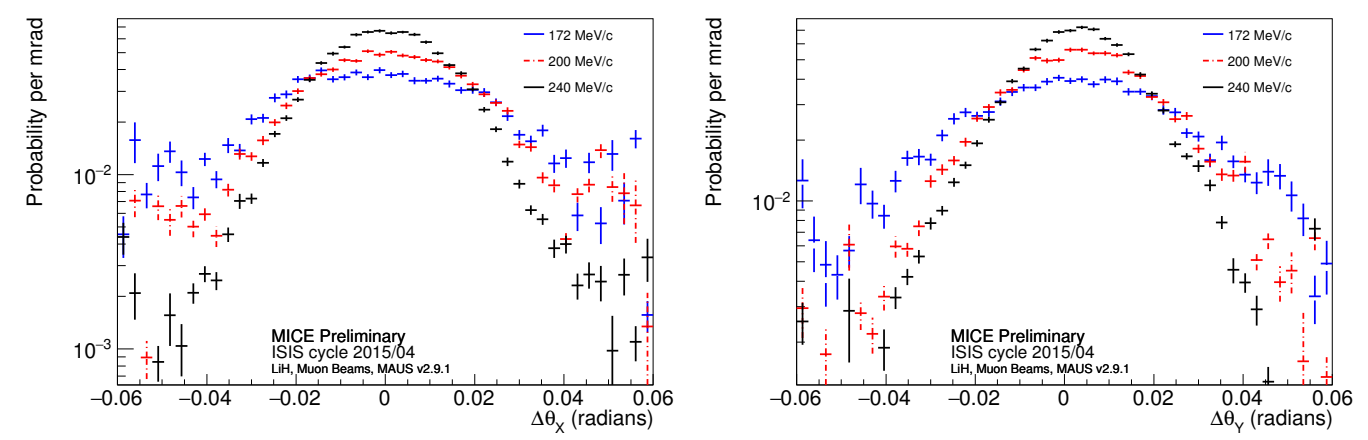

Figure 5: The results of the scattering analysis using data from all three nominal beam settings. Scattering widths are reported after application of deconvolution.

The scattering in the absorber material is the physical quantity of interest. To extract this information the effects of scattering in non absorber materials and detector resolution that will appear in the overall scattering measurement must be deconvolved from the required scattering distribution. A deconvolution algorithm using Bayesian statistics [26] has been used based on the implementation contained in the RooUnfold package [27]. This method uses the simulation to provide a probability of observing a given scattering angle from the trackers for a given true 

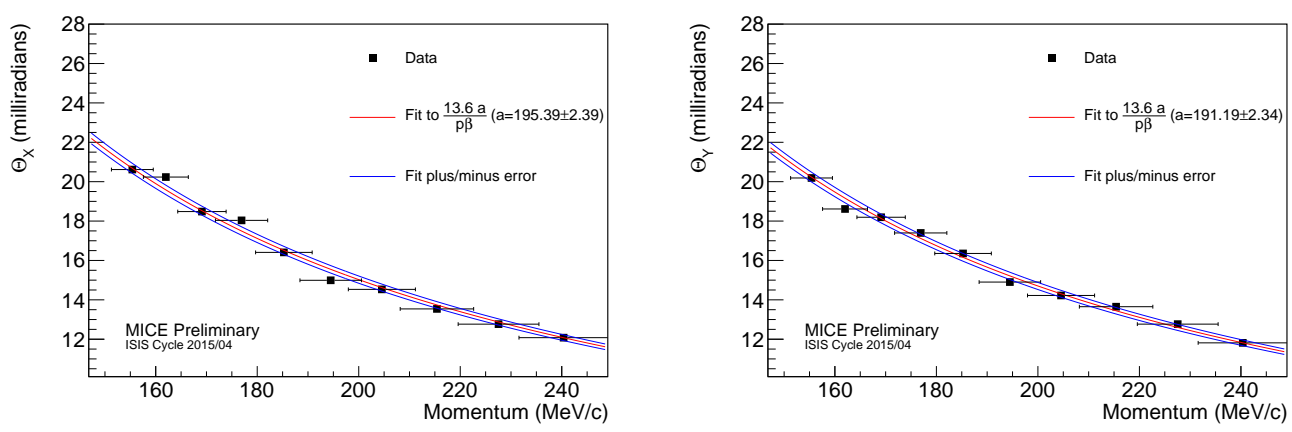

Figure 6: The analysis was repeated for each momentum window scanning over the full MICE data set. The results are shown in the $X$ projection (left) and the $Y$ projection (right)

scattering angle in the absorber, $P\left(\Delta \Theta_{j}^{\text {tracker }} \mid \Delta \Theta_{i}^{\text {abs }}\right)$. This conditional probability is then used to estimate the number of particles that experience an absorber scattering angle,

$$
n\left(\theta_{i}^{\text {abs }}\right)=\sum_{j=1}^{n_{E}} n\left(\theta_{j}^{\text {tracker }}\right) P\left(\theta_{i}^{\text {abs }} \mid \theta_{j}^{\text {tracker }}\right)
$$

which requires the calculation of the conditional probability

$$
P\left(\theta_{i}^{\text {abs }} \mid \theta_{j}^{\text {tracker }}\right)=\frac{P\left(\theta_{j}^{\text {tracker }} \mid \theta_{i}^{\text {abs }}\right) P_{0}\left(\theta_{i}^{\text {abs }}\right)}{\sum_{l=1}^{n_{c}} P\left(\theta_{j}^{\text {tracker }} \mid \theta_{l}^{\text {abs }}\right) P_{0}\left(\theta_{l}^{\text {abs }}\right)}
$$

The estimate is refined through multiple applications of the algorithm by updating the prior probability by letting $P_{0}\left(\theta_{i}^{a b s}\right)=n\left(\theta_{i}^{a b s}\right) / \sum_{i=1}^{n_{c}} n\left(\theta_{i}^{a b s}\right)$ in iterations subsequent to the initial calculation in which a flat prior is used. The conditional probability $P\left(\theta_{j}^{\text {tracker }} \mid \theta_{i}^{a b s}\right)$ is derived from the convolution where $\theta^{\text {tracker }}$ is drawn from the sum of the reconstructed scattering angle in the empty absorber data and the scattering angle in the absorber from the convolution model, and $\theta_{i}^{a b s}$ is the scattering angle in the absorber alone. The final scattering distributions at each of the nominal momentum points are shown in figure 5 and over the full momentum range in 6 .

\section{Conclusion}

MICE has measured multiple Coulomb scattering off a lithium hydride target for muons with momentum between 140 and $240 \mathrm{MeV} / \mathrm{c}$. These data have been compared to popular simulation packages such as GEANT4 and other relevant models such as Moliere and Carlisle-Cobb. A study of the systematics is in progress with a MICE publication currently being prepared. Future work will including a measurement of multiple Coulomb scattering off liquid hydrogen, a measurement of scattering with magnetic field in the cooling channel and an energy loss measurement.

\section{References}

[1] Y. Fukuda et al. Evidence for oscillation of atmospheric neutrinos. Phys.Rev.Lett., 81:1562-1567, 1998. 
[2] Q. R. Ahmad et al. Direct evidence for neutrino flavor transformation from neutral current interactions in the Sudbury Neutrino Observatory. Phys. Rev. Lett., 89:011301, 2002.

[3] S. Geer. Neutrino beams from muon storage rings: Characteristics and physics potential. Phys. Rev., D57:6989-6997, 1998.

[4] Diktys Stratakis, Richard C. Fernow, J. Scott Berg, and Robert B. Palmer. Tapered channel for six-dimensional muon cooling towards micron-scale emittances. Phys. Rev. ST Accel. Beams, 16(9):091001, 2013.

[5] Diktys Stratakis and Robert B. Palmer. Rectilinear six-dimensional ionization cooling channel for a muon collider: A theoretical and numerical study. Phys. Rev. ST Accel. Beams, 18(3):031003, 2015.

[6] Diktys Stratakis. A hybrid six-dimensional muon cooling channel using gas filled rf cavities. JINST, 12(09):P09027, 2017.

[7] M. Bogomilov et al. The MICE Muon Beam on ISIS and the beam-line instrumentation of the Muon Ionization Cooling Experiment. JINST, 7:P05009, 2012.

[8] R. Bertoni et al. The design and commissioning of the MICE upstream time-of-flight system. Nucl. Instrum. Meth., A615:14-26, 2010.

[9] Lucien Cremaldi, David A. Sanders, Peter Sonnek, Donald J. Summers, and Jim Reidy, Jr. A Cherenkov Radiation Detector with High Density Aerogels. IEEE Trans. Nucl. Sci., 56:1475-1478, 2009.

[10] M. Adinolfi, A. Aloisio, F. Ambrosino, A. Andryakov, A. Antonelli, et al. Calibration and reconstruction performances of the KLOE electromagnetic calorimeter. Nucl.Instrum.Meth., A461:344-347, 2001.

[11] D. Adams et al. Electron-Muon Ranger: performance in the MICE Muon Beam. JINST, 10(12):P12012, 2015.

[12] K. A. Olive et al. Review of Particle Physics. Chin. Phys., C38:090001, 2014.

[13] Gerald R. Lynch and Orin I. Dahl. Approximations to multiple Coulomb scattering. Nucl. Instrum. Meth., B58:6-10, 1991.

[14] Timothy Carlisle. Step IV of the Muon Ionization Cooling Experiment (MICE) and the multiple scattering of muons. DPhil thesis, University of Oxford, 2013.

[15] D. Attwood et al. The scattering of muons in low Z materials. Nucl. Instrum. Meth., B251:41-55, 2006.

[16] Bruno Rossi and Kenneth Greisen. Cosmic-ray theory. Rev. Mod. Phys., 13:240-309, 1941.

[17] G. Moliere. Theory of the scattering of fast charged particles. 2. Repeated and multiple scattering. $Z$. Naturforsch., A3:78-97, 1948.

[18] H. A. Bethe. Moliere's theory of multiple scattering. Phys. Rev., 89:1256-1266, 1953.

[19] William T. Scott. The theory of small-angle multiple scattering of fast charged particles. Rev. Mod. Phys., 35:231-313, 1963.

[20] S. Agostinelli et al. GEANT4: A Simulation toolkit. Nucl. Instrum. Meth., A506:250-303, 2003.

[21] Laszlo Urban. A model for multiple scattering in Geant4. 2006. CERN-OPEN-2006-077.

[22] V. N. Ivanchenko, O. Kadri, M. Maire, and L. Urban. Geant4 models for simulation of multiple scattering. J. Phys. Conf. Ser., 219:032045, 2010. 
[23] T. Carlisle, J. Cobb, and D. Neuffer. Multiple Scattering Measurements in the MICE Experiment. Conf. Proc., C1205201:1419-1421, 2012.

[24] D. Adams et al. Characterisation of the muon beams for the Muon Ionisation Cooling Experiment. Eur. Phys. J., C73:2582, 2013.

[25] M. Bogomilov et al. Pion Contamination in the MICE Muon Beam. JINST, 11(03):P03001, 2016.

[26] G. D’Agostini. A Multidimensional unfolding method based on Bayes' theorem. Nucl. Instrum. Meth., A362:487-498, 1995.

[27] Tim Adye. Unfolding algorithms and tests using RooUnfold. In Proceedings of the PHYSTAT 2011 Workshop, CERN, Geneva,Switzerland, January 2011, CERN-2011-006, pages 313-318, 2011. 